\title{
Mid-Infrared Computational Spectroscopy with an Electrically-Tunable Graphene Metasurface
}

\author{
Vivek Raj Shrestha ${ }^{1}$, Benjamin Craig, ${ }^{1}$, Matin Amani ${ }^{2,3}$, James Bullock ${ }^{2,3,4}$, Ali Javey ${ }^{2,3}$, and Kenneth B. \\ Crozier ${ }^{1,4}$ \\ 1 School of Physics, University of Melbourne, VIC 3010, Australia \\ 2 Electrical Engineering and Computer Sciences, University of California, Berkeley, Berkeley, CA 94720, USA \\ 3 Materials Sciences Division, Lawrence Berkeley National Laboratory, Berkeley, CA 94720, USA \\ 4 Department of Electrical and Electronic Engineering, University of Melbourne, VIC 3010, Australia \\ Corresponding Author e-mail address: kenneth.crozier@unimelb.edu.au
}

\begin{abstract}
We demonstrate graphene-plasmonic metasurfaces whose mid-infrared reflection spectra are electrically-tunable. Using measurements of the power reflected by the metasurfaces at different drive voltages, the source spectrum is computationally reconstructed by the recursive least squares method.

OCIS codes: (230.4110) Modulators; (300.6340) Spectroscopy, infrared; (080.1753) Computation methods
\end{abstract}

\section{Introduction}

Mid-infrared $(\sim 3-12 \mu \mathrm{m})$ spectroscopy is important in fields that include environmental monitoring and food safety testing [1]. Miniaturized mid infrared spectrometers would be advantageous for in-field applications not requiring the very high resolution that can be achieved by traditional Fourier-transform infrared (FTIR) spectrometers. The latter are frequently bulky and expensive. Miniaturized spectrometers have been demonstrated that comprise arrays of passive (i.e. non-tunable) filters matched to detector arrays. These filters have included Fabry-Perot etalons [2], quantum dots [3], and plasmonic filters [4,5]. One of the drawbacks is that many filters (e.g. 100) are needed for high resolution reconstruction. Replacing the passive filters with dynamically tuned filters presents an opportunity to substantially reduce the number of filters needed, thereby enabling further miniaturization. Devices with tunable resonances have been reported that combine graphene with nanoantennas [6,7], but have not been employed for spectral reconstruction. Here, we demonstrate a dynamically-tunable device that, when combined with a detector array, would permit the realization of an ultra-compact mid-infrared spectrometer. Our device consists of three electrically tunable-graphene metasurfaces, each comprising metallic nanostructures integrated with graphene and having an extent of $30 \mu \mathrm{m} \times 30 \mu \mathrm{m}$. The reflectance spectrum of each metasurface can be controlled by applying a gate voltage $\left(\mathrm{V}_{\mathrm{g}}\right)$. Our work demonstrates that even though the device contains only three metasurfaces, their electrical tunability means that this is sufficient for spectral reconstruction of a mid-infrared light source. This is done by measuring the reflection spectra and total power reflected by each metasurface at different values of $V_{g}$. The measured data is input to a recursive least square (RLS, [8]) algorithm, which estimates the incident spectrum.

\section{Design and Fabrication}

Figure 1a shows the schematic of our metasurface, which comprises cross shaped nanoantennas over a single layer of graphene, separated from a metallic reflector (that also functions as a back gate) by a $\mathrm{TiO}_{2}$ spacer. The structural parameters of the device include the nanoantenna period (P), width (W), and gap (G), and the thickness (t) of the $\mathrm{TiO}_{2}$ cavity layer. Finite difference time domain (FDTD) simulations were performed to choose these parameters to produce a reflection dip in the mid infrared regime. Simulated reflection spectra for a device with parameters $\mathrm{P}=2250 \mathrm{~nm}, \mathrm{G}=60 \mathrm{~nm}$ and $\mathrm{W}=200 \mathrm{~nm}$ are shown in Fig $1 \mathrm{~b}$. In this device, there is also an $\mathrm{Al}_{2} \mathrm{O}_{3}$ layer ( $30 \mathrm{~nm}$ thick) between graphene and $\mathrm{TiO}_{2}$, included to improve gate performance. It can be seen that the spectral position of the dip is shifted substantially as the chemical potential of the graphene was varied from 0.1 to $0.2 \mathrm{eV}$.

To fabricate the devices, a CVD graphene monolayer was transferred onto a highly doped silicon wafer predeposited with $\mathrm{Au}\left(120 \mathrm{~nm}\right.$ thick), $\mathrm{TiO}_{2}\left(800 \mathrm{~nm}\right.$ thick) and $\mathrm{Al}_{2} \mathrm{O}_{3}(30 \mathrm{~nm}$ thick) layers. Three devices with $\mathrm{P}=1200$, 1500 and $2250 \mathrm{~nm}$ were fabricated by e-beam lithography, e-beam evaporation $(5 \mathrm{~nm}$ Ti and $25 \mathrm{~nm} \mathrm{Au})$ and lift off. Rectangles of graphene were patterned by e-beam lithography and dry etching (oxygen plasma).

\section{Results and Discussion}

We measured reflection spectra of the fabricated device at different $\mathrm{V}_{\mathrm{g}}$ with an FTIR microscope system (Spotlight 200i, Perkin Elmer). To normalize the results, reflection spectra were measured from a reference sample comprising a gold film. Reflection spectra $\operatorname{Rvg}_{\mathrm{g}}(\lambda)$ of the three devices were measured at 21 different values of $\mathrm{V}_{\mathrm{g}}$ ranging from $10 \mathrm{~V}$ to $12 \mathrm{~V}$ in the steps of $1 \mathrm{~V}$ (Fig. $1 \mathrm{c}-\mathrm{e}$ ), where the wavelength of the reflectance dip (i.e. the resonance wavelength) of those devices was seen to be tuned in the range of $\lambda=8.3$ to $9.2 \mu \mathrm{m}$. We also determined the spectrum 
$S(\lambda)$ of light reflected from each device at each $\mathrm{V}_{\mathrm{g}}$. This represents $S(\lambda)=g(\lambda) \times M C T_{\text {resp }}(\lambda) \times \mathrm{R}_{\mathrm{Vg}}(\lambda)$, where $g(\lambda)$ is power density spectrum of the light source of our FTIR system (silicon carbide globar) and $M C T_{\text {resp }}(\lambda)$ is responsivity of the mercury cadmium telluride (MCT) detector of the FTIR microscope. For each measurement, we integrated $S(\lambda)$ over $\lambda=4.6 \mu \mathrm{m}$ to $10 \mu \mathrm{m}$, which yields a column vector $(69 \times 1)$ that mimics what would be measured by a spectrometer consisting of a detector integrated with the filter array. This column vector was input, along with $\operatorname{Rvg}_{\mathrm{Vg}}(\lambda)$, to our RLS algorithm to estimate $g(\lambda) \times M C T_{\text {resp }}(\lambda)$, i.e. the spectrum of our globar multiplied by the detector responsivity. The results were in good agreement with the globar spectrum directly measured with the FTIR microscope as seen in Fig. 1f.

In summary, we have demonstrated dynamic control of mid-infrared light by tuning the resonance of a graphene integrated metasurface and used the same to demonstrate spectral reconstruction of a globar source.

(a)

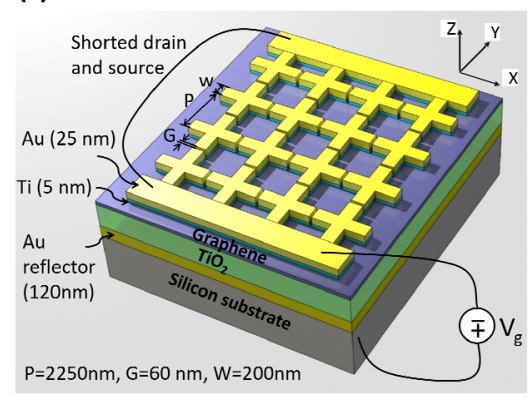

(d)

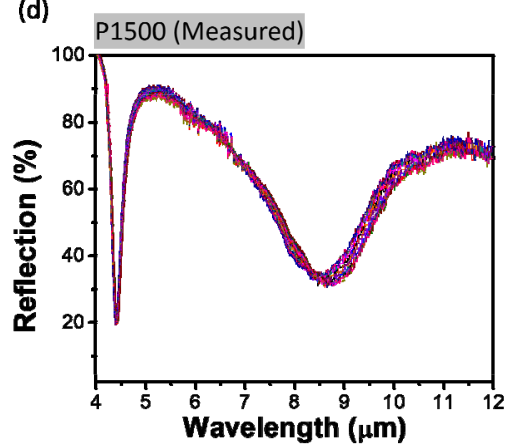

(b)
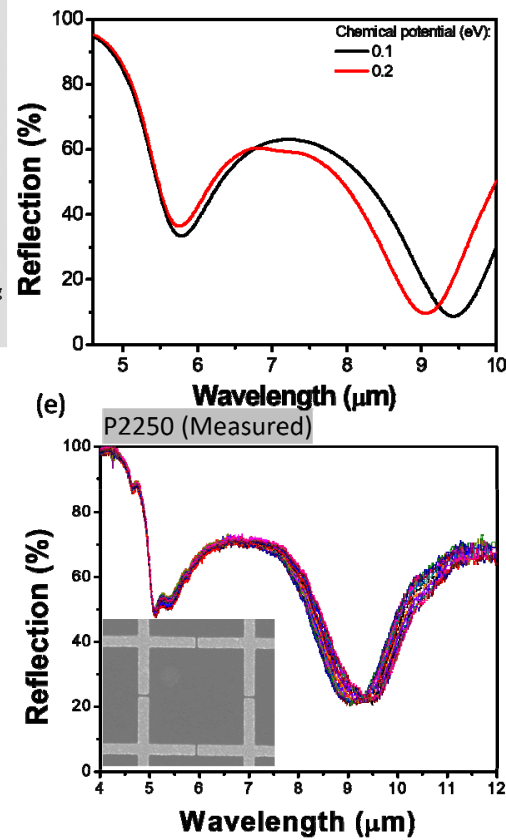

(c)

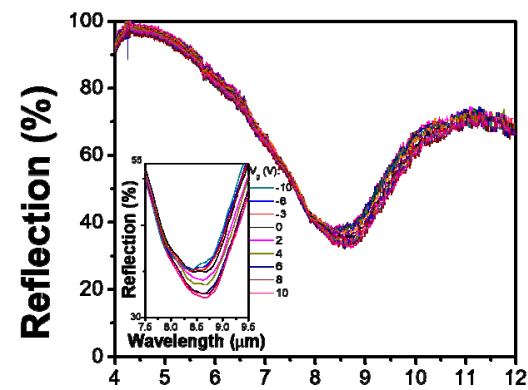

(f)

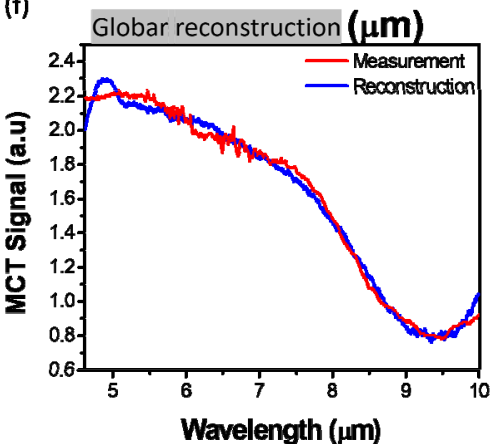

$V_{g}(V):$

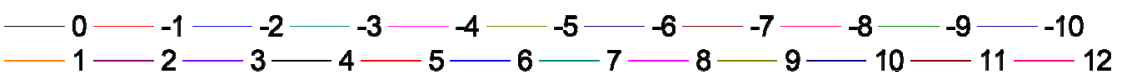

Fig. 1. (a) Schematic of metasurface device with back gate. (b) Simulated reflection spectra of device for different graphene chemical potentials Measured reflection spectra for fabricated devices with $P=(\mathrm{c}) 1200 \mathrm{~nm}$; Inset: Magnified image of reflection spectra (d) $1500 \mathrm{~nm}$ and (e) 2250 $\mathrm{nm}$ at different $\mathrm{V}_{\mathrm{g}}$; Inset: SEM image of the fabricated antennas (f) Reconstruction of MCT signal of globar source (i.e. $g(\lambda) \times M C T_{\text {resp }}(\lambda)$ ) over a wavelength range of $4.6 \mu \mathrm{m}$ to $10 \mu \mathrm{m}$, with 1917 data points.

\section{References}

[1] Y. Li, et al., "Surface-enhanced molecular spectroscopy (SEMS) based on perfect-absorber metamaterials in the mid-infrared," Sci. Rep. 3, 2865 (2013).

[2] E. Huang et al., "Etalon Array Reconstructive Spectrometry," Sci. Rep. 7, 40693 (2017).

[3]. J. Bao et al., "A colloidal quantum dot spectrometer Nature," 523, 67 (2015).

[4] B. Craig et al., "Experimental demonstration of infrared spectral reconstruction using plasmonic metasurfaces," Opt. Lett. 43, 4481-4484 (2018).

[5] M. Najiminaini et al., "Nanohole-array-based device for 2D snapshot multispectral imaging," Sci. Rep. 3, 2589 (2013).

[6] Y. Yao et al., "Electrically tunable metasurface perfect absorbers for ultrathin mid-infrared optical modulators," Nano Lett. 14, 6526-6532 (2014).

[7] Y. Yao et al., "Wide wavelength tuning of optical antennas on graphene with nanosecond response time," Nano Lett. 14, 214-219 (2014).

[8] M. H. Hayes, "9.4: Recursive Least Squares," in Statistical Digital Signal Processing and Modelling, Wiley, 1996, p.541.

Performed in part at Melbourne Centre for Nanofabrication (MCN) in Victorian Node of Australian National Fabrication Facility.Supported in part by DARPA (HR0011-16-1-004), by VESKI, and by the Australian Research Council (DP150103736 and FT140100577). The content of this paper does not necessarily reflect the position or policy of the United States Government, and no official endorsement should be inferred. 


\section{University Library}

\section{- M M I N E R VA A gateway to Melbourne's research publications}

Minerva Access is the Institutional Repository of The University of Melbourne

\section{Author/s:}

Shrestha, VR;Craig, B;Amani, M;Bullock, J;Javey, A;Crozier, KB

Title:

Mid-infrared computational spectroscopy with an electrically-tunable graphene metasurface

\section{Date:}

2019-01-01

Citation:

Shrestha, V. R., Craig, B., Amani, M., Bullock, J., Javey, A. \& Crozier, K. B. (2019). Midinfrared computational spectroscopy with an electrically-tunable graphene metasurface. CLEO: Science and Innovations 2019, Part F129-CLEO_SI 2019, OSA - Optical Society of America. https://doi.org/10.1364/CLEO_SI.2019.SW3J.2.

Persistent Link:

http://hdl.handle.net/11343/294880 